\title{
ON THE SIMPLICITY OF HOMEOMORPHISM GROUPS OF A TILABLE LAMINATION
}

\author{
JOSÉ ALISTE-PRIETO AND SAMUEL PETITE
}

\begin{abstract}
We show that the identity component of the group of homeomorphisms that preserve all leaves of a $\mathbb{R}^{d}$ - tilable lamination is simple. Moreover, in the one dimensional case, we show that this group is uniformly perfect. We obtain a similar result for a dense subgroup of homeomorphisms.
\end{abstract}

\section{INTRODUCTION}

In this paper it is shown that the connected component of the identity of the

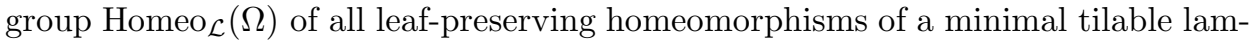
ination $\Omega$ in any dimension is a simple group. We also prove that this group is equal to the group of homeomorphisms that are isotopic to the identity and that is open in $\operatorname{Homeo}_{\mathcal{L}}(\Omega)$.

Similar results were obtained in the 60's by G. Fisher [7 for the group of all homeomorphisms of a closed topological manifold of dimension smaller or equal than three. The algebraic simplicity for groups of homeomorphisms and diffeomorphisms of manifolds has been widely studied in the literature: In 1961, R. Anderson [2], generalizing the work of G. Fisher [7, showed the group of stable homeomorphisms of a manifold is simple. Later, D. Epstein [6] established sufficient conditions on a group of homeomorphisms, for the commutator subgroup to be simple. This means that a group satisfying Epstein's conditions is simple if and only if it is perfect (i.e. its commutators subgroup is the whole group).

It is also worth mentioning the works of M. Herman [9, W. Thurston 18 and J. Mather 12 who provided a nearly complete classification for the simplicity of diffeomorphism groups on manifolds.

Given a smooth foliation $\mathcal{F}$ over a manifold $M$, T. Rybicki [15] and T. Tsuboi [16 studied the simplicity and perfectness of the identity component of the group $G_{\mathcal{F}}(M)$ of all leaf preserving diffeomorphisms of $(M, \mathcal{F})$. Notice here that these groups do not satisfy Epstein's conditions.

On the other hand, tilable laminations have been recently introduced as a geometric model for the study of non-periodic tilings [3. They also appear as suspensions of minimal Cantor $\mathbb{Z}^{d}$-actions, like minimal subshifts. In addition, they include some classical laminated spaces as the dyadic solenoid. These spaces are locally homeomorphic to the product of an open set in $\mathbb{R}^{d}$ and a Cantor set. In other words, these are laminated spaces with a Cantor transversal. They are also

1991 Mathematics Subject Classification. 57S05, 37C85 .

Key words and phrases. leaf preserving homeomorphisms, tilable lamination.

J. Aliste-Prieto acknowledges financial support from Fondecyt Iniciación 11121510 and Anillo DySyRf ACT-1103. S. Petite acknowledges financial support from the ANR SUBTILE 0879. This work is part of the program MathAmSud DYSTIL 12Math-02. 
endowed with a natural $\mathbb{R}^{d}$-action, which we we call the translation flow. Like in the case of foliations of manifolds, groups of homeomorphisms on tilable laminations in general do not satisfy Epstein's conditions.

We denote by $D(\Omega)$ the group of deformations, that is, $D(\Omega)$ is the path connected component of the identity in $\operatorname{Homeo}(\Omega)$ endowed with the $C^{0}$-topology. We conjointly consider the following class of homeomorphisms, which arises naturally in the context of non-periodic tilings, namely the group of homeomorphisms preserving the vertical structure (see the precise definition in Section 2 ) denoted $\operatorname{Homeo}_{v s p}(\Omega)$. Roughly speaking, such homeomorphisms map any small Cantor transversal into a Cantor transversal. Notice that $\operatorname{Homeo}_{v s p}(\Omega)$ is a dense subgroup of $\operatorname{Homeo}_{\mathcal{L}}(\Omega)$. Let $D_{v s p}(\Omega)$ denote the path connected component of the identity in $\operatorname{Homeo}_{v s p}(\Omega)$. Our aim is to show the simplicity of these groups.

Another motivation for studying these groups comes from topological orbit equivalence theory: two tilable laminations $\Omega_{1}, \Omega_{2}$ are orbit equivalent if there is a homeomorphism between the spaces mapping any orbit onto an orbit. Because of the totally disconnected transversal structure, $\Omega_{1}$ and $\Omega_{2}$ are orbit equivalent if and only if they are homeomorphic. A difficult result of Rubin 14 asserts that the group $\operatorname{Homeo}(\Omega)$ is a complete invariant of the orbit equivalence class of the lamination $\Omega$ : Any algebraic group isomorphism of these groups is induced by an homeomorphism on the topological laminations. It follows that the group $D(\Omega)$ is an invariant of flow equivalence.

For a topological group $G$, we denote by $G^{0}$ the connected component of the identity in $G$.

Theorem 1.1. Let $\Omega$ be a minimal tilable lamination. Let $G$ be either $\operatorname{Homeo}_{\mathcal{L}}(\Omega)$ or $\operatorname{Homeo}_{v s p}(\Omega)$. Then,

(1) $\operatorname{Homeo}_{\mathcal{L}}^{0}(\Omega)=D(\Omega)$ and $\operatorname{Homeo}_{v s p}^{0}(\Omega)=D_{v s p}(\Omega)$;

(2) $G^{0}$ is open in $G$;

(3) $G^{0}$ is simple.

Moreover, when the translation flow is expansive, e.g. for tiling spaces, the connected component of the identity in $\operatorname{Homeo}(\Omega)$ is the group of deformations.

Proposition 1.2. Let $\Omega$ be a minimal tilable lamination. If the translation flow is expansive, then the identity component $\operatorname{Homeo}^{0}(\Omega)$ is equal to $D(\Omega)$ and is open in $\operatorname{Homeo}(\Omega)$.

The proof of Theorem 1.1 follows the same strategy as in 7 for the triangulated manifolds (see 4 for a recent survey). In the next section we recall basic properties of tilable laminations and their homeomorphisms. By using a generalization of the Schoenflies Theorem due to R. Edwards and R. Kirby, we show, in Section 3 , the groups under consideration satisfy the partition property (called also fragmentation property), and we prove Proposition 1.2 and the items (1), (2) of Theorem 1.1. We give in Section 4 a sufficient condition for a commutator subgroup of $\operatorname{Homeo}_{\mathcal{L}}(\Omega)$ to be simple. Next, we prove in Section 5 that the groups $\operatorname{Homeo}_{\mathcal{L}}(\Omega)$ and $\operatorname{Homeo}_{v s p}(\Omega)$ are perfect and we conclude the proof of Theorem 1.1 with the main result of Section 4. In the last section, we show, for the one-dimensional case, that these groups are uniformly perfect: more precisely, any element can be written as a product of two commutators in the group. This last result is similar to [8] for $C^{\infty}$ leaf preserving diffeomorphisms of $C^{\infty}$ foliations. 


\section{Preliminaries}

2.1. Background on tilable lamination. We recall here some basic properties of tilable laminations and we refer to $[3$ for a more detailed exposition. Let $\Omega$ be a compact metric space. Assume that there exist a cover of $\Omega$ by open sets $U_{i}$ and homeomorphisms called charts $h_{i}: U_{i} \rightarrow D_{i} \times C_{i}$ where $C_{i}$ is a topological space and $D_{i}$ is an open set of $\mathbb{R}^{d}$. These open sets and charts define an atlas of a flat lamination if the transition maps $h_{j} \circ h_{i}^{-1}$ read on their domains of definition

$$
h_{i, j}(t, c)=\left(t+a_{i, j}, \gamma_{i, j}(c)\right),
$$

where the $a_{i, j}$ 's are elements of $\mathbb{R}^{d}$ and the maps $\gamma_{i, j}$ are continuous. Two atlases are equivalent if their union is also an atlas.

A flat lamination is the data of a compact metric space $\Omega$ together with an equivalence class of atlases $\mathcal{L}$. A box is the domain of a chart in the maximal atlas of $\mathcal{L}$. For any point $x$ in a box $B$ with coordinates $\left(t_{x}, c_{x}\right)$ in the chart $h$, the set $h^{-1}\left(D \times\left\{c_{x}\right\}\right)$ is called the slice and the set $h^{-1}\left(\left\{t_{x}\right\} \times C\right)$ is called the vertical of $x$ in $B$. Since a transition map transforms slices into slices and verticals into verticals, these definitions make sense. As usual, a leaf of $\Omega$ is the smallest connected set that contains all the slices it intersects. From (11), it is clear that each leaf is a manifold with a flat Riemannian metric.

Definition 2.1. A tilable lamination $(\Omega, \mathcal{L})$ (or a $\mathbb{R}^{d}$-solenoid) is a flat lamination such that

- every leaf of $\mathcal{L}$ is isometric to $\mathbb{R}^{d}$.

- There exists a transversal $\Xi$ (a compact subset of $\Omega$ such that for any leaf $L$ of $\mathcal{L}, L \cap \Xi$ is non empty and a discrete subset with respect the manifold topology of the leaf $L$ ) which is a Cantor set.

For short, we will speak about $\Omega$ as a tilable lamination when there is no confusion. If every leaf is dense in $\Omega$, we say that the lamination is minimal. By (11), the action by translations on $\mathbb{R}^{d}$ can be transported to a local action (also by translations) along the slices. In fact, these local translations induce a continuous and free $\mathbb{R}^{d}$-action $T$ over $\Omega$, see [3] for details. We refer to this action as the translation flow over $\Omega$. To simplify the notations we write $\omega-t:=T(t, \omega)$ for $\omega$ in $\Omega$ and $t$ in $\mathbb{R}^{d}$. It is easy to see that the leaves of the lamination coincide with the orbits of the translation flow. Again by (1), the canonical orientation on $\mathbb{R}^{d}$ induce an orientation on each leaf of $\Omega$. Given a box $B$ that reads $h^{-1}(D \times C)$ in a chart $h$, by identifying a vertical in $B$ with the Cantor set $C$, we can write $B=T(C, D)=C-D$, thus avoiding the explicit reference to the chart $h$.

Basic examples of minimal tilable laminations are given by the suspensions of minimal $\mathbb{Z}^{d}$ action on a Cantor set with locally constant ceiling functions. The tilable lamination structure also appears in the dynamical systems associated to non-periodic repetitive tilings and Delone sets of the Euclidean space, see [3]. In these examples, the translation flow is expansive in the following sense (see [13]).

Definition 2.2. Let $\eta>0$. The translation flow of a tilable lamination $\Omega$ is said to be $\eta$-expansive if when one has points $x, y \in \Omega$ and a homeomorphism $h: \mathbb{R}^{d} \rightarrow \mathbb{R}^{d}$ satisfying $h(0)=0$ and $d(x-t, y-h(t))<\eta$ for all $t \in \mathbb{R}^{d}$, then there must exist $t_{0} \in B_{\eta}(0)$ such that $x-t_{0}=y$.

The translation flow is said expansive, if it is $\eta$-expansive for some constant $\eta$. 
This last property will allow us to show, in the next section, that any homeomorphism that is close enough to the identity must by leaf-preserving.

A box in $\Omega$ is said to be internal if its closure is included in another box of $\Omega$. In all the rest of the paper, any box will be supposed to internal. An internal box $B$ is said to be of ball type if it can be written as $B=C-D$, where $D$ is an open ball in $\mathbb{R}^{d}$. Instead, if $D$ is a $d$-cube $\left(a_{1}, b_{1}\right) \times \cdots \times\left(a_{d}, b_{d}\right)$ in $\mathbb{R}^{d}$, then $B$ is said to be a box of cubic type. In this case, and if $f$ is a $\ell$-face $(0 \leq \ell \leq d)$ of the cube $D$, then the set $C-f$ is said to be an $\ell$-vertical boundary of $B$. A box cover of $\Omega$ is a cover $\left\{B_{i}\right\}_{i}$ of $\Omega$, where each $B_{i}$ is a box. Box covers of ball type and cubic type are defined in the same way.

Definition 2.3. A collection of boxes $\mathcal{B}=\left\{B_{i}\right\}_{i=0}^{t}$ in $\Omega$ is a box decomposition, if the following assertions hold:

(1) the $B_{i}$ 's are pairwise disjoint,

(2) the closures of the $B_{i}$ 's form a cover of $\Omega$.

Also, if the boxes $B_{i}$ are of cubic type, then $\mathcal{B}$ is a box decomposition of cubic type.

Box decompositions were introduced in [3] as a tool in the study of tilable laminations. The key lemma (see bellow) asserts that any box cover of cubic type can be turned into a box decomposition of cubic type. It follows that every tilable lamination admits a box decomposition of cubic type.

Lemma 2.4 (3]). Let $\Omega$ be a tilable lamination and $\left\{B_{i}\right\}_{i=0}^{t}$ be a box cover of cubic type of $\Omega$. Then, there exists a box decomposition of cubic type $\mathcal{B}^{\prime}=\left\{B_{i}^{\prime}\right\}_{i=0}^{n}$, such that, for all $i$, whenever $B_{i}^{\prime}$ intersects $B_{j}$ for some $j$, then it is included in $B_{j}$.

The union of all the $\ell$-vertical boundaries $(0 \leq \ell \leq d)$ of all the boxes of a box decomposition of cubic type $\mathcal{B}$ is called the $\ell$-skeleton of $\mathcal{B}$.

2.2. Homeomorphisms of tilable laminations. Let $\Omega$ be a tilable lamination and denote by $\operatorname{Homeo}(\Omega)$ the set of homeomorphisms of $\Omega$. We endow it with the $C^{0}$-topology, which is induced by the distance

$$
\delta(f, g)=\sup _{x \in \Omega} d(f(x), g(x))+\sup _{x \in \Omega} d\left(f^{-1}(x), g^{-1}(x)\right), \quad f, g \in \text { Homeo }(\Omega) .
$$

The support of a homeomorphism $f$ in $\operatorname{Homeo}(\Omega)$ is defined by

$$
\operatorname{supp} f=\overline{\{x \in \Omega \mid f(x) \neq x\}} .
$$

It is easy to see that $\operatorname{supp} f$ is $f$-invariant and $\operatorname{supp} \phi f \phi^{-1}=\phi(\operatorname{supp} f)$ for every $\phi \in \operatorname{Homeo}(\Omega)$.

Since the verticals of a tilable lamination $\Omega$ are totally disconnected, the pathconnected components coincide with the leaves of the lamination. Thus, every element of $\operatorname{Homeo}(\Omega)$ maps each leaf onto a (possibly different) leaf. We define $\operatorname{Homeo}_{\mathcal{L}}(\Omega)$ be the group of all leaf-preserving homeomorphisms of $\Omega$. Recall that a homeomorphism $f$ of $\Omega$ is homotopic to the identity, if there exists a continuous map $F:[0,1] \times \Omega \rightarrow \Omega$ such that $F(0, \cdot)=I d$ and $F(1, \cdot)=f$. If, in addition, $F(t, \cdot)$ is a homeomorphism of $\Omega$ for each $t \in \Omega$, then we say that $f$ is isotopic to the identity or a deformation. The set $D(\Omega)$ denotes the group of all the deformations. Clearly, homeomorphisms that are homotopic to the identity belong to $\mathrm{Homeo}_{\mathcal{L}}(\Omega)$. If $\Omega$ is minimal, then the converse is also true. 
Theorem 2.5 (11, [11). Let $\Omega$ be a minimal tilable lamination. Then every $f \in$ $\operatorname{Homeo}_{\mathcal{L}}(\Omega)$ is homotopic to the identity. In particular, for every $f \in \operatorname{Homeo}_{\mathcal{L}}(\Omega)$, there is a continuous map $\Phi_{f}: \Omega \rightarrow \mathbb{R}^{d}$, called the displacement of $f$, which is uniquely defined by the equation

$$
f(\omega)=\omega-\Phi_{f}(\omega) \quad \text { for all } \omega \in \Omega .
$$

We say the displacement of $f$ is smaller than $\varepsilon$ when $\|\Phi\|_{\infty}<\varepsilon$.

When the translation flow is expansive, we get the following refinement.

Proposition 2.6. Let $\Omega$ be tilable lamination. Suppose its translation flow is $\eta$ expansive. Then, $\operatorname{Homeo}_{\mathcal{L}}(\Omega)$ is open in $\operatorname{Homeo}(\Omega)$.

Proof. Define $B=\{f \in \operatorname{Homeo}(\Omega) \mid \delta(f, I d)<\eta\}$ and take any $f \in B$. Since the translation flow is free and homeomorphisms map leaves onto leaves, for every $\omega \in \Omega$ there is a continuous map $h: \mathbb{R}^{d} \rightarrow \mathbb{R}^{d}$ such that $f(\omega)-s=f(\omega-h(s))$ for all $s \in \mathbb{R}^{d}$.

Thus,

$$
d(f(\omega)-s, \omega-h(s)) \leq \delta(f, I d)<\eta \quad \text { for all } s \in \mathbb{R}^{d} .
$$

It follows from the expansivity of $\Omega$ that there exists a $t_{0} \in \mathbb{R}^{d}$ such that $f(\omega)=$ $\omega-t_{0}$. Since $\omega$ was arbitrary, this means that $f$ preserves each leaf and thus $f \in \operatorname{Homeo}_{\mathcal{L}}(\Omega)$, which means that $B \subset \operatorname{Homeo}_{\mathcal{L}}(\Omega)$. The fact that $\operatorname{Homeo}_{\mathcal{L}}(\Omega)$ is open now follows from a standard argument.

Corollary 2.7. If the translation flow on the tilable lamination $\Omega$ is expansive, then $\operatorname{Homeo}^{0}(\Omega)$ is open and

$$
\operatorname{Homeo}^{0}(\Omega)=\operatorname{Homeo}_{\mathcal{L}}^{0}(\Omega) .
$$

Proof. Since the connected component is the greatest connected set containing the identity, we have $\operatorname{Homeo}_{\mathcal{L}}^{0}(\Omega) \subset \operatorname{Homeo}^{0}(\Omega)$. By Proposition 2.6 and the connexity property, we get $\operatorname{Homeo}^{0}(\Omega) \subset \operatorname{Homeo}_{\mathcal{L}}(\Omega)$ and so $\operatorname{Homeo}^{0}(\Omega) \subset \operatorname{Homeo}_{\mathcal{L}}^{0}(\Omega)$, which concludes the proof.

In the context of laminations arising from the study of non-periodic tilings, an important class of homeomorphisms is given by homeomorphisms with the following property.

Definition 2.8. A homeomorphism $f \in \operatorname{Homeo}(\Omega)$ preserves the vertical structure if, given a point $x$ in a vertical $C$ of a box $B$ and a vertical $C^{\prime}$ of a box $B^{\prime}$ containing $f(x)$, then there is a clopen subset $\tilde{C} \subset C$ containing $x$ such that for every $y \in \tilde{C}$, $f(y) \in C^{\prime}$.

Alternatively, provided that $\Omega$ is minimal, a map $f \in \operatorname{Homeo}_{\mathcal{L}}(\Omega)$ preserves the vertical structure if and only if its deplacement $\Phi$ is transversally locally constant. In the context of non-periodic repetitive tilings, this notion corresponds to the notion of strong pattern-equivariance (see [10]) of the map $t \mapsto \Phi_{f}(\omega-t)$ for any fixed $\omega \in \Omega$. We denote by $\operatorname{Homeo}_{v s p}(\Omega)$ the collection of homeomorphisms preserving the vertical structure. It is plain to check that $\operatorname{Homeo}_{v s p}(\Omega)$ is dense in $\operatorname{Homeo}(\Omega)$. We will denote by $D_{v s p}(\Omega)$ the path-connected component of the identity in $\operatorname{Homeo}_{v s p}(\Omega)$. 


\section{PARTition PROPERTY}

Definition 3.1. A group $G$ of homeomorphisms of $\Omega$ satisfies the partition property if for every box cover $\left\{B_{i}\right\}_{i=0}^{t}$ of $\Omega$, and for any $f \in G$, there exists a decomposition $f=g_{1} \cdots g_{\ell}$ where $g_{i} \in G$ and $\operatorname{supp} g_{i} \subset B_{j(i)}$ for $i=1, \ldots, \ell$.

In this section, following [7] and using the box decomposition structure of tilable laminations we show:

Proposition 3.2. Let $\Omega$ be a minimal tilable lamination. The two groups $\operatorname{Homeo}_{\mathcal{L}}^{0}(\Omega)$ and $\mathrm{Homeo}_{v s p}^{0}(\Omega)$ satisfy the partition property.

We will also show assertions (1) and (2) of Theorem 1.1. To prove this result, we will use several lemmas. We start by showing that every map having its support included in a box of ball type is a deformation.

Lemma 3.3. Let $\Omega$ be a minimal tilable lamination and $B$ be a box of ball type. Any map $g \in \operatorname{Homeo}_{\mathcal{L}}(\Omega)$ (resp. in $\operatorname{Homeo}_{v s p}(\Omega)$ ), with support in the interior of $B$ is a deformation of the identity (resp. $g \in D_{v s p}(\Omega)$ ).

Proof. The proof is classical by using the Alexander's trick. We can assume that the closure $\bar{B}$ of the box reads $h^{-1}(D \times C)$ in a chart $h$, with $D$ a closed ball in $\mathbb{R}^{d}$ of radius $r>0$ centered at the origin. Since the support of the map $g$ is in $B$, the map $g$ preserves any slice of the box $B$. So, for any $c \in C$, let $g_{c}: D \rightarrow D$ be the map defined by $g\left(h^{-1}(t, c)\right)=h^{-1}\left(g_{c}(t), c\right)$ for $t \in D$. Now, for any $t \in[0,1]$, let $F_{t}: D \times C \rightarrow D \times C$ be the map

$$
F_{t}(x, c)= \begin{cases}\left((1-t) g_{c}\left(\frac{x}{1-t}\right), c\right) & \text { if }|x|<r(1-t) \\ (x, c) & \text { if }|x| \geq r(1-t) .\end{cases}
$$

It is plain to check the map $h^{-1} F_{t} h$ gives an isotopy between the identity and the map $g$.

In the case where $g \in \operatorname{Homeovsp}_{v}(\Omega)$, up to subdivide the clopen set $C$, we can assume that the map $g_{c}$ is independent of $c$. Hence the isotopy is also in Homeo $v s p(\Omega)$.

Given two subsets $A \subset B$ of a topological space $X$, an embedding $f: A \rightarrow B$ is a continuous and injective map. This embedding is proper if $f^{-1}(\partial B)=A \cap \partial B$. The next theorem says that any proper embedding of a neighborhood of a compact set $K$ into a ball, sufficiently close to the identity, can be isotoped to an embedding which is the identity on $K$. Moreover the isotopy depends continuously of the embedding. This theorem, true in any dimension, generalizes a version of the Schoenflies Theorem.

Theorem 3.4. 5] Let $D$ be a (closed or open) ball in $\mathbb{R}^{d}, K \subset D$ a compact subset and $U$ a neighborhood of $K$ in $D$. Then, for any proper embedding $f: U \rightarrow D$ close enough of the identity (for the $C^{0}$ topology), there exists a continuous map $H: U \times[0,1] \rightarrow D$ such that:

- For any $t \in[0,1], H(\cdot, t): U \rightarrow D$ is a proper embedding.

- $H(\cdot, 0)=f(\cdot)$ and $H(\cdot, 1)_{\mid K}=I d_{\mid K}$.

- There is a compact neighborhood $K_{2}$ of $K$ in $U$, such that for any $t \in[0,1]$, $H(\cdot, t)_{\mid U \backslash K_{2}}=f(\cdot)_{\mid U \backslash K_{2}}$.

- $H$ depends continuously on $f$ for the $C^{0}$ topology. 
Applied in our context, a first consequence is that any map close to the identity can be interpolated by a map with a support in a box.

Lemma 3.5. Let $\Omega$ be a tilable lamination, let $B$ be a box of ball type and $B^{\prime}$ be a box with closure included in $B$. Then there exists an $\varepsilon>0$ such that for any homeomorphism $f \in \operatorname{Homeo}_{\mathcal{L}}(\Omega)$ (resp. $\operatorname{Homeo}_{v s p}(\Omega)$ ) with a displacement smaller than $\varepsilon$, there exists a map $g \in \operatorname{Homeo}_{\mathcal{L}}(\Omega)$ (resp. Homeo vsp $\left.(\Omega)\right)$ with $\operatorname{supp} g \subset B$ such that $g_{\mid B^{\prime}}=f_{\mid B^{\prime}}$. Moreover, the displacement of $g$ depends continuously on $f$ for the $C^{0}$-topology.

Proof. Without loss of generality, we may assume that $B$ reads $h^{-1}\left(D_{3} \times C\right)$ in a chart $h$ with $D_{3}$ a ball in $\mathbb{R}^{d}$. Assume that $D_{1} \times C$ is a compact neighborhood of $h\left(B^{\prime}\right)$ with $D_{1} \subset D_{3}$ a compact subset and let $D_{2} \subset D_{3}$ be a neighborhood of $D_{1}$.

We consider a map $f \in \operatorname{Homeo}_{\mathcal{L}}(\Omega)$ with a displacement smaller than $\varepsilon$ (defined later). By continuity, for a small enough $\varepsilon$, the set $f\left(h^{-1}\left(D_{2} \times C\right)\right)$ is in $B$, and for any $c \in C, f\left(h^{-1}\left(D_{2} \times\{c\}\right)\right) \subset h^{-1}\left(D_{3} \times\{c\}\right)$. For any $c \in C$, let $f_{c}: D_{2} \rightarrow$ $D_{3}$ be the embedding defined by $f_{c}(\cdot)=\left(h f h^{-1}\right)(\cdot, c)$ and let $K$ be a compact neighborhood of $\partial D_{2}$ proper in $U=D_{2} \backslash D_{1}$. For an $\varepsilon$ small enough and for any $c \in C$, Theorem 3.4 applied to the maps $f_{c}: U \rightarrow D_{3}$, gives us embeddings $h_{c}=H_{c}(\cdot, 1): U \rightarrow D_{3}$. We define then the maps $\bar{f}_{c}: D_{3} \rightarrow D_{3}$ by $\bar{f}_{c \mid U}:=h_{c}$ and $\bar{f}_{c \mid D_{1}}:=f_{c \mid D_{1}}$ and $\bar{f}_{c \mid D_{3} \backslash D_{2}}:=I d_{\mid D_{3} \backslash D_{2}}$ Let $\bar{f}: B \rightarrow B$ be the map defined by $\bar{f} \circ h^{-1}(t, c)=h \circ\left(\bar{f}_{c}(t), c\right)$ for any $(t, c) \in D_{3} \times C$. By construction, $\bar{f}$ is a homeomorphism, $\bar{f}_{\mid h^{-1}\left(D_{1} \times C\right)}=f_{\mid h^{-1}\left(D_{1} \times C\right)}$ and $\bar{f}_{\mid \partial B}=I d_{\mid \partial B}$. So $\bar{f}$ can be extended by the identity to all the tilable lamination $\Omega$ to define a homeomorphism. This gives the map $g$.

Here again, when $f \in \operatorname{Homeo}_{v s p}(\Omega)$, up to subdividing the clopen set $C$, we can assume that the map $f_{c}$ is independent of $c$. So the same is true for $\bar{f}$ and it belongs to $\operatorname{Homeo}_{v s p}(\Omega)$.

Proposition 3.6. Let $\Omega$ be a minimal tilable lamination and $\mathcal{B}=\left\{B_{i}\right\}_{i=1}^{k}$ be a box cover of $\Omega$. Then, there are $\varepsilon>0$ and an integer $\ell>0$ such that for every $f \in \operatorname{Homeo}_{\mathcal{L}}(\Omega)$ (resp. in $\operatorname{Homeo}_{v s p}(\Omega)$ ) with displacement smaller than $\varepsilon$, there exists a decomposition $f=g_{1} \cdots g_{\ell}$ with $g_{i} \in \operatorname{Homeo}_{\mathcal{L}}(\Omega)\left(\right.$ resp. $\left.\operatorname{Homeo}_{v s p}(\Omega)\right)$ and $\operatorname{supp} g_{i} \subset B_{j(i)}$.

Proof. Let $\mathcal{B}^{\prime}=\left\{B_{0}^{\prime}, B_{1}^{\prime}, \ldots, B_{m}^{\prime}\right\}$ be the box decomposition of cubic type given by Lemma 2.4. Up to subdividing each box $B_{i}^{\prime}$ into smaller boxes, we can assume that the closure of every box $B_{i}^{\prime}$ is included in a box $B_{j(i)}$. We will construct, by induction on $0 \leq i \leq d$, a homeomoprhism $f_{i}$ which equals the identity on a neighborhood of the $i$-skeleton of $\mathcal{B}^{\prime}$ and equals $f$ outside. At each step, we use Lemma 3.5 to approximate $f_{i-1}$ by maps with support in a small box.

For any 0-vertical boundary $V$ of a box $B_{i}^{\prime}$, let $B_{V}^{(0)}$ be a box containing $V$ in its interior and included in a box $B_{j(i)}$. Let $B_{1}^{(0)}, \ldots, B_{n}^{(0)}$ be the collection of these boxes containing all the 0 -vertical boundaries. Up to refine the boxes $B_{i}^{(0)}$, we can assume that they are pairwise disjoint. The union of all theses boxes $B_{i}^{(0)}$ covers the 0 -skeleton of $\mathcal{B}^{\prime}$.

Step 0. Applying Lemma 3.5 to any box $B_{i}^{(0)}$ and any neighborhood of the 0vertical $V \cap B_{i}^{(0)}$ (when not empty), we get, for an $\varepsilon$ small enough, a $g_{i} \in \operatorname{Homeo}_{\mathcal{L}}(\Omega)$ with supp $g_{i} \subset B_{i}^{(0)}$ such that $g_{i}=f$ on a neighborhood of $V \cap B_{i}^{(0)}$. It follows 
that the maps $g_{1}, \ldots, g_{n}$ commute; and $f_{0}=g_{1}^{-1} \circ \cdots \circ g_{n}^{-1} \circ f$ is the identity in a neighborhood $U_{0}$ of the 0 -skeleton of $\mathcal{B}^{\prime}$. Moreover the displacement of $f_{0}$ continuously depends on the displacement of $f$.

Step i. $1 \leq i \leq d-1$. Let us assume that $f_{i} \in \operatorname{Homeo}_{\mathcal{L}}(\Omega)$ equals the identity on a neighborhood $U_{i-1}$ of the $i-1$-skeleton of $\mathcal{B}^{\prime}$. We do the same as for the former step. Let $B_{1}^{(i)}, \ldots, B_{n_{i}}^{(i)}$ be a collection of boxes such that any $i$-vertical boundary $V$ of $\mathcal{B}^{\prime}$ is in the interior of a box $B_{j}^{(i)} \subset B_{t(j)}$. Up to refine the boxes $B_{j}^{(i)}$, we may assume that the sets $B_{j}^{(i)} \backslash U_{i-1}$ are pairwise disjoint. Applying Lemma 3.5 to any box $B_{j}^{(i)}$ and to a neighborhood of the $i$-vertical $\left(V \backslash U_{i-1}\right) \cap B_{j}^{(i)}$ (when not empty), we have, for an $\varepsilon$ small enough, a $g_{j}^{(i)} \in \operatorname{Homeo}_{\mathcal{L}}(\Omega)$ with $\operatorname{supp} g_{j}^{(i)} \subset B_{j}^{(i)}$ such that $g_{j}^{(i)}=f$ on a neighborhood of $\left(V \backslash U_{i-1}\right) \cap B_{j}^{(i)}$. We get that the maps $g_{1}^{(i)}, \ldots, g_{n_{i}}^{(i)}$ commute; and $f_{i}=\left(g_{1}^{(i)}\right)^{-1} \circ \cdots \circ\left(g_{n_{i}}^{(i)}\right)^{-1} \circ f_{i-1}$ is the identity in a neighborhood of the $i$-skeleton.

Hence the homeomorphism $f_{d-1}$ preserves each box $B_{i}^{\prime}$, and $f_{d-1}$ can be written as the composition of homeomorphisms with support in each box of the decomposition $\mathcal{B}^{\prime}$. Moreover if $f \in \operatorname{Homeo}_{v s p}(\Omega)$, then $f_{d-1} \in \operatorname{Homeo}_{v s p}(\Omega)$ also. This proves the proposition.

The following proposition shows the assertions (1) and (2) of Theorem 1.1 .

Proposition 3.7. Let $\Omega$ be a minimal tilable lamination. Then, $D(\Omega)$ is open in $\operatorname{Homeo}_{\mathcal{L}}(\Omega)$ and $D(\Omega)=\operatorname{Homeo}_{\mathcal{L}}^{0}(\Omega)$. Similarly, $D_{v s p}(\Omega)$ is open in $\operatorname{Homeo}_{v s p}(\Omega)$ and $D_{v s p}(\Omega)=$ Homeo $_{v s p}^{0}(\Omega)$.

Proof. Let $\mathcal{B}=\left\{B_{i}\right\}_{i=1}^{k}$ be a box cover of ball type of $\Omega$. Let $\rho$ be the Lebesgue number of $\mathcal{B}$ and consider an atlas $\mathcal{B}^{\prime}=\left\{B_{j}^{\prime}\right\}_{j=1}^{\ell}$ of $\Omega$ such that the diameter of any box $B_{j}^{\prime}$ is smaller than $\rho$. Then by Proposition 3.6, any map $f \in \operatorname{Homeo}_{\mathcal{L}}(\Omega)$ with displacement small enough, can be written as a product of maps $g_{i} \in \operatorname{Homeo}_{\mathcal{L}}(\Omega)$ with support in a $B_{j(i)}^{\prime}$. Thus by Lemma 3.3 we get that any $g_{i}$ is in $D(\Omega)$, and finally $f \in D(\Omega)$. This means that the identity lies in the interior of $D(\Omega)$. Standard arguments on topological groups show then that $D(\Omega)$ is open and closed in $\operatorname{Homeo}_{\mathcal{L}}(\Omega)$ and $D(\Omega)=\operatorname{Homeo}_{\mathcal{L}}^{0}(\Omega)$. The proof is similar for the group Homeo $v s p(\Omega)$.

Finally, we can obtain the proof of Proposition 3.2

of Proposition 3.2. Let $H$ be either $\operatorname{Homeo}_{\mathcal{L}}^{0}(\Omega)$ or $\operatorname{Homeo}_{v s p}^{0}(\Omega)$ and let $\mathcal{B}$ be an atlas of $\Omega$. Proposition 3.7 gives us a constant $\eta>0$ such that any element of $\operatorname{Homeo}_{\mathcal{L}}(\Omega)$ (resp. in $\operatorname{Homeo}_{v s p}(\Omega)$ ) with a displacement smaller than $\eta$ is in $D(\Omega)$ (resp. $D_{v s p}(\Omega)$ ). Up to refining the covering $\mathcal{B}$, we can assume that every element of $\mathcal{B}$ has a diameter smaller than $\eta$. Since the group $H$ is connected, it is enough to show the partition property for any $f \in H$ with an arbitrary small displacement $\varepsilon$. By taking the $\varepsilon$ given by Proposition 3.6. we can write $f$ as a product of elements $g_{i}$ in $\operatorname{Homeo}_{\mathcal{L}}(\Omega)$ with displacement smaller than $\eta$. By Proposition 3.7, we get $g_{i} \in H$ and this shows the partition property.

\section{Simplicity of the commutator subgroup}

Epstein's result [6] asserts that if a group of homeomorphism is factorizable and acts transitively on open sets, then its commutator subgroup is simple. Let $\Omega$ be a minimal tilable lamination and let $G$ be a subgoup of $\operatorname{Homeo}_{\mathcal{L}}^{0}(\Omega)$. In general, we 
cannot expect $G$ to act transively on open sets. We need to replace the transivity condition with another one which is more adapted to laminated spaces. Thus, we give here, a sufficient condition on a subgroup of $\operatorname{Homeo}_{\mathcal{L}}^{0}(\Omega)$ so that the derived subgroup is simple.

Theorem 4.1. Let $\Omega$ be a minimal $\mathbb{R}^{d}$ tilable lamination. Let $G \subset \operatorname{Homeo}_{\mathcal{L}}^{0}(\Omega)$ be a group such that:

i) $G$ satisfies the partition property.

ii) For any boxes $B_{1}=C-D_{1}, B_{2}=C-D_{2}$ of ball type whose closures lie in a box $B=C-K$, there exists a $g \in G^{\prime}$ such that $B_{2} \subset g\left(B_{1}\right)$.

Then the derived group $G^{\prime}=[G, G]$ is simple.

Proof. Let $N$ be a non-trivial normal subgroup of $G^{\prime}$. We have to show that $N=G^{\prime}$.

Lemma 4.2. There exists an atlas $\mathcal{B}$ of the solenoid $\Omega$ such that for every box $B$ of $\mathcal{B}$ there is a map $n_{B} \in N$ such that $B$ and $n_{B}(B)$ are disjoint.

Proof. Let $I d \neq n \in N$. There is a box $B_{0}=C_{0}-D_{0}$ of ball type such that $B_{0}$ and $n\left(B_{0}\right)$ are disjoint. Since the translation flow is free and minimal and translations have the vertical structure preserving property, it follows that for every $x \in \Omega$, there is a clopen subset $C_{x} \subset C_{0}$ and an open ball $D_{x} \subset \mathbb{R}^{d}$ with $D_{0} \subset D_{x}$ such that $C_{x}-D_{x}$ is a box of $\Omega$ containing the point $x$.

Let $\tilde{B}_{x}$ be a box included in $C_{x}-D_{x}$ containing the point $x$. By hypothesis ii), there is a $g \in G^{\prime}$ such that $\tilde{B}_{x} \subset g\left(C_{x}-D_{0}\right)$. It is then straightforward to check that the box $\tilde{B}_{x}$ is disjoint from its image by the map $g \circ n \circ g^{-1} \in N$. The collection of boxes $\left\{\tilde{B}_{x}\right\}_{x \in \Omega}$ satisfies the condition of the statement.

Let $\mathcal{B}$ be the finite cover given by Lemma 4.2 of the tilable lamination $\Omega$ by boxes and let $\rho>0$ be its Lebesgue number. Let us recall that for any ball of radius $\rho$ in $\Omega$ there exists a box of $\mathcal{B}$ containing this ball. Let $\mathcal{B}_{1}$ be a box cover of cubic type of $\Omega$, equivalent to $\mathcal{B}$, and such that any box has a diameter smaller than $\rho$. It follows that when two boxes $B_{1}, B_{2}$ of $\mathcal{B}_{1}$ are intersecting, there exists a box $B$ of $\mathcal{B}$ containing $B_{1} \cup B_{2}$.

The following is an algebraic lemma due to T. Tsuboi [17].

Lemma 4.3 ([17, Lemma 3.1]). Let $B$ be a box and $n$ be an homeomorphism such that $n(B) \cap B=\emptyset$. Then for any homeomorphisms $a, b \in G$ with supports in $B$, the commutator $[a, b]$ can be written as a product of 4 conjugates of $n$ and $n^{-1}$.

Proof. Let $h=n^{-1} a n$, since the supports are disjoint, we have $h b=b h$. So we get

$$
\begin{aligned}
a b a^{-1} b^{-1} & =n h n^{-1} b n h^{-1} n^{-1} b^{-1} \\
& =n h n^{-1} h^{-1} h b n h^{-1} b^{-1} b n^{-1} b^{-1} \\
& =n\left(h n^{-1} h^{-1}\right)\left(b h n h^{-1} b^{-1}\right)\left(b n^{-1} b^{-1}\right) .
\end{aligned}
$$

Now, for each $B \in \mathcal{B}_{1}$, let $G_{B}$ be the subgroup of $G$ of homeomorphisms with support in $B$, and let $H$ be the subgroup of $G$ generated by all the $G_{B}$ with $B \in \mathcal{B}_{1}$. By the partition property (item i)), the groups $H$ and $G$ are the same. It is well-known that the commutator subgroup of $H$ is generated by the conjugates of commutators of elements in a generating set of $H$. So to prove the theorem, we 
just have to show for any boxes $B_{1}, B_{2}$ in $\mathcal{B}_{1}$, and for $f_{1} \in G_{B_{1}}$ and $f_{2} \in G_{B_{2}}$, that the commutator $\left[f_{1}, f_{2}\right]$ belongs to $N$.

If the boxes $B_{1}$ and $B_{2}$ do not intersect, then every point of $\Omega$ is fixed by either $f_{1}$ or $f_{2}$, which means that $\left[f_{1}, f_{2}\right]=i d$ and thus belong to $N$.

Suppose now that $B_{1}$ and $B_{2}$ intersect and let $B$ be a box in $\mathcal{B}$ containing $B_{1} \cup B_{2}$. By Lemma 4.2, there exists a $n \in N$ such that $B \cap n(B)=\emptyset$. Thus by Lemma 4.3, we have $\left[f_{1}, f_{2}\right] \in N$, and then $H^{\prime}=N$.

\section{Perfectness}

To show that the groups $\operatorname{Homeo}_{\mathcal{L}}^{0}(\Omega)$ and $\operatorname{Homeo}_{s}^{0}(\Omega)$ are simple, we will first prove in this section they are perfect. For this, we need the next lemma stating a transitivity of the action of the group $D_{v s p}(\Omega)$ on specific boxes of a same box. This is a reinforcement of condition ii) in Theorem 4.1. We will deduce then the perfectness. This will imply, together with the partition property, that these groups satisfy the conditions of Theorem 4.1, and henceforth they are simple.

Lemma 5.1. Let $B_{1}=C-D_{1}$ and $B_{2}=C-D_{2}$ be two boxes of ball type, and let $B_{0}=C-V$ be a box containing the closures of $B_{1}$ and $B_{2}$. Then there exists a $g \in D_{v s p}(\Omega)$ such that $B_{2}=g\left(B_{1}\right)$.

Proof. Let $h$ be the chart associated to the box $C-V$. The boxes $B_{1}$ and $B_{2}$ read respectively $h^{-1}\left(D_{1} \times C\right)$ and $h^{-1}\left(D_{2} \times C\right)$, with $D_{1}, D_{2}$ two balls in $V$. Up to composing with a translation $T_{\rho}$, we may assume that $D_{1} \subset D_{2} \subset V$ or $D_{2} \subset D_{1} \subset V$. In both cases, it is straightforward to construct a homeomorphism $\psi \in D_{v s p}(\Omega)$ with support in $C-V$ such that $\left.\psi\left(h^{-1}\left(D_{1}\right) \times C\right)\right)=h^{-1}\left(D_{2} \times C\right)$.

The proof of the next theorem follows directly the ideas of Frédéric Le Roux (see Theorem 1.1.3 in 4 for a proof on a surface). On a manifold, this shows directly that the group of homeomorphism is simple. Here, because of the lack of homogeneity, it enables just to show the groups $\operatorname{Homeo}_{\mathcal{L}}^{0}(\Omega)$ and $\operatorname{Homeo}_{v s p}^{0}(\Omega)$ are perfect.

Theorem 5.2. For $\Omega$ a minimal $\mathbb{R}^{d}$-tilable lamination, the groups $\operatorname{Homeo}_{\mathcal{L}}^{0}(\Omega)$ and $\mathrm{Homeo}_{v s p}^{0}(\Omega)$ are perfect.

Proof. Let $H$ denotes either $\operatorname{Homeo}_{\mathcal{L}}^{0}(\Omega)$ or $\operatorname{Homeo}_{v s p}^{0}(\Omega)$. We have to show that any element of $H$ can be written as a commutators product. It is simple to find two non commuting elements $a, b \in D_{v s p}(\Omega)$ with supports in a box $B \subset \Omega$ of ball type. So the element $g=[a, b] \in H$ is not the identity. We will show that $N(g)$, the normal subgroup generated by $g$, contains all the elements $f$ of $H$ with support in the box $B$. Recall that a conjugate of a commutator is still a commutator, it will follow that $f$ can be written as a finite product of commutators. Since the box $B$ is arbitrary, we get the conclusion by the partition property (Proposition 3.2).

We have $g \neq I d$, so we consider a box $B^{\prime} \subset B$ such that $g\left(B^{\prime}\right)$ and $B^{\prime}$ are disjoint. In a chart $h$, we may assume that $B^{\prime}$ reads $h^{-1}\left(B_{r}(0) \times C\right)$ where $B_{r}(0)$ denotes the Euclidean ball in $\mathbb{R}^{d}$ of radius $r>0$ centered at 0 . For any integer $n \geq 0$, we define a nested sequence of boxes $B_{n}:=h^{-1}\left(B_{r / 2^{n+1}}(0) \times C\right)$. It is simple to construct an element $\psi$ of $D_{v s p}(\Omega)$ with support in $B^{\prime}$, such that $\psi\left(B_{n}\right)=B_{n+1}$ of $n \geq 0$. We get then that the homeomorphism $k=[\psi, g] \in N(g)$ satisfies $k\left(B_{n}\right)=B_{n+1}$ for $n \geq 0$ and $\operatorname{supp} k \subset B^{\prime} \cup g\left(B^{\prime}\right)$ ( $k$ is the product of $\psi$ and $g \psi^{-1} g^{-1}$ that have disjoint supports). 
Let $A_{n}=B_{n} \backslash B_{n+1}$ and let us show that $N(g)$ contains all the element of $H$ with support in $A_{1}$. For any $\phi \in H$ with a support in $A_{1}$, we claim that $\phi k$ and $k$ are conjugate: notice, we have $k=\phi^{-1}(\phi k) \phi$ on $A_{0}, \bigsqcup_{n \geq 0} A_{n}=\bigsqcup_{n \geq 0} g^{n}\left(A_{0}\right)=$ $\bigsqcup_{n>0}(\phi k)^{n}\left(A_{0}\right)$ and $k_{\mid B \backslash B_{0}}=\phi k_{\mid B \backslash B_{0}}$; It is then standard to check that the continuous homeomorphism $\tilde{\phi}$ defined by

$$
\tilde{\phi}_{\mid A_{n}}:=(\phi k)^{n} \phi k_{\mid A_{n}}^{-n} \text { for any } n \geq 0 \quad \text { and } \quad \tilde{\phi}_{\mid \Omega \backslash B_{0}}=I d,
$$

can be extended by continuity to $\overline{\bigcup_{n>0} A_{n}}=B_{0}$, is in $H$ and satisfies $k=\tilde{\phi}^{-1}(\phi k) \tilde{\phi}$ on $\Omega$. We get then $\phi k \in N(k) \subset N(g)$, so $\phi \in N(g)$.

Finally, let $f \in H$ with a support in $B$. By Lemma 5.1 there exists a $\phi \in H$ such that the support of $\phi f \phi^{-1}$ is in $A_{1}$. So by the last result we have $f \in N(g)$.

We have then the groups $\operatorname{Homeo}_{\mathcal{L}}^{0}(\Omega)$ and $\operatorname{Homeo}_{v s p}^{0}(\Omega)$ equal their commutator groups. So by Lemma 5.1 and Theorem 4.1, we get the main result: Theorem 1.1,

\section{UNIFORM PERFECTNESS IN DIMENSION ONE}

Theorem 5.2 asserts that any homeomorphism of a tilable lamination $\Omega$ is a product of commutators. For the one dimension, we can be more precise.

Theorem 6.1. For $\Omega$ a minimal $\mathbb{R}$-tilable lamination, any element of $\operatorname{Homeo}_{\mathcal{L}}^{0}(\Omega)$ (resp. $D_{v s p}(\Omega)$ ) can be written as a product of two commutators of $\operatorname{Homeo}_{\mathcal{L}}^{0}(\Omega)$ (resp. $\left.D_{v s p}(\Omega)\right)$.

Before proving this theorem, we need some technical lemmas. The first one solves the problem of perfectness for homeomorphisms with support in a box. Recall that any map $f \in \operatorname{Homeo}_{\mathcal{L}}^{0}(\Omega)$ preserves the orientation, so, if $\Phi$ denotes its displacement, for any $\omega \in \Omega$, the map $\mathbb{R} \ni t \mapsto t+\Phi(\omega-t) \in \mathbb{R}$ is increasing.

Lemma 6.2. Let $\Omega$ be a minimal $\mathbb{R}$-tilable lamination and let $f \in \operatorname{Homeo}_{\mathcal{L}}^{0}(\Omega)$ (resp. Homeo ${ }_{v s p}^{0}(\Omega)$ ) with support included in a box B. Then there exists a homeomorphism $g \in \operatorname{Homeo}_{\mathcal{L}}^{0}(\Omega)$ (resp. $\operatorname{Homeo}_{\text {vsp }}^{0}(\Omega)$ ) with support in $B$ such that $g f g^{-1}=f^{2}$. In particular $f=[g, f]$.

Proof. Since $f$ preserves the orientation, it preserves each slice of the box $B=C-I$, with $C$ a clopen set and $I$ an interval. For any $x \in C$, we denote by $f_{x}: I \rightarrow I$ the increasing map induced on the slice of $x$ : i.e. defined by $f_{x}(t)=t+\Phi(x-t)$ so that $f(x-t)=x-f_{x}(t)$ for any $t \in I$.

For any $z_{0} \in\{z \in B ; f \neq I d\}$, let the vertical $C_{z_{0}}$ be $C-t_{0}$ where $z_{0}$ writes $x_{0}-t_{0}$ with $x_{0} \in C, t_{0} \in I$. We define the local strip

$$
V_{z_{0}}:=\left\{x-t ; x \in C, t_{0} \leq t<f_{x}\left(t_{0}\right)\right\} .
$$

By the definition, the sets $\left\{f^{n}\left(V_{z_{0}}\right)=V_{f^{n}\left(z_{0}\right)}\right\}_{n \in \mathbb{Z}}$ are pairwise disjoints, and $\cup_{n \in \mathbb{Z}} f^{n}\left(V_{z_{0}}\right)$ is a $f$-invariant open set. Hence there exist a collection at most countable of points $\left\{z_{n}\right\}_{n \geq 0} \subset\{f \neq I d\}$ and local strips $V_{n}=V_{z_{n}}$ such that

$$
\operatorname{supp} f=\cup_{n \geq 0} \overline{\cup_{p \in \mathbb{Z}} f^{p}\left(V_{n}\right)} \text { and the sets }\left\{\cup_{p \in \mathbb{Z}} f^{p}\left(V_{n}\right)\right\}_{n \geq 0} \text { are pairwise disjoint. }
$$

Notice that since $f$ preserves the orientation, a point is fixed by $f$ if and only it is a fixed point of $f^{2}$. So we have $\operatorname{supp} f=\operatorname{supp} f^{2}$. 
For each $n \geq 0, z_{n}=x_{n}-t_{n}$ with $t_{n} \in I, x_{n} \in C$, let $h_{n}:\left[t_{n}, f_{z_{n}}\left(t_{n}\right)\right) \rightarrow$ $\left[t_{n}, f_{z_{n}}^{2}\left(t_{n}\right)\right)$ be the bijective affine map fixing $t_{n}$. It is then straightforward to check that the continuous map $g_{n}$ defined on $\cup_{p \in \mathbb{Z}} f^{p}\left(V_{n}\right)$ by

$$
g_{n \mid f^{p}\left(V_{n}\right)}:=f^{2 p} \circ h_{n} \circ f^{-p},
$$

can be continuously extended by the identity to $\partial \cup_{p \in \mathbb{Z}} f^{p}\left(V_{n}\right)$ and satisfies $f^{2} \circ g_{n}=$ $g_{n} \circ f$ where it is defined. Hence we can define a homeomorphism $g$ on $\Omega$ with support in $B$ such that $g_{\mid \cup_{p \in \mathbb{Z}} f^{p}\left(V_{n}\right)}=g_{n}$ for every $n \geq 0$. Notice furthermore that $g$ is in $\operatorname{Homeo}_{v s p}^{0}(\Omega)$ when $f$ is.

The next lemma, is a version of lemma 3.5 without the condition to be close of the identity.

Lemma 6.3. Let $\Omega$ be a minimal $\mathbb{R}$-tilable lamination and let $f \in \operatorname{Homeo}_{\mathcal{L}}^{0}(\Omega)$ (resp. Homeo ${ }_{v s p}^{0}(\Omega)$ ). Suppose that $B^{\prime}=C-J$ and $B=C-I$ are boxes of cubic type such that the closure of $f\left(B^{\prime}\right) \cup B^{\prime}$ is contained in $B$. Then, there exists a $g$ in $D(\Omega)$ (resp. $\left.D_{v s p}(\Omega)\right)$ with support contained in $B$ such that $\left.f\right|_{B^{\prime}}=\left.g\right|_{B^{\prime}}$.

Proof. Without loss of generality, we may assume that $I, J$ are two open intervals such that $0 \in \bar{J} \subset I$. Consider $\eta: I \rightarrow[0,1]$ a continuous function that is equal to zero on the boundary of $I$, is equal to one on $J$ and affine on each component of $I \backslash J$. Let $\phi$ be the displacement function of $f$, and define $\psi: \Omega \rightarrow \mathbb{R}$ by

$$
\psi(x-t)=\eta(t) \phi(x-t) \quad \text { for any } x \in C, t \in I,
$$

and by zero on the complement of $B$. It is clear that $\psi$ is a continuous function. Thus, $g(x):=x-\psi(x)$ is continuous and coincides with $f$ on $B^{\prime}$, and since $J \subset I$, it is also increasing by the choice of $\eta$. It is plain to check $g \in D(\Omega)$.

Lemma 6.4. Let $\Omega$ ba a minimal $\mathbb{R}$-tilable lamination and let $f \in \operatorname{Homeo}_{\mathcal{L}}^{0}(\Omega)$ (resp. Homeo $v_{v s p}^{0}(\Omega)$ ). Then there exist two boxes $B^{\prime} \subset B$ and two homeomorphisms $f_{1}, f_{2} \in \operatorname{Homeo}_{\mathcal{L}}^{0}(\Omega)$ (resp. Homeo $v_{v s p}^{0}(\Omega)$ ) such that

- $\operatorname{supp} f_{2} \subset B$;

- $\left.f_{1}\right|_{B^{\prime}}=\left.I d\right|_{B^{\prime}}$;

- $f=f_{1} \circ f_{2}$.

Proof. Let $x$ be a point of $\Omega$. The points $x$ and $f(x)$ are in the same leaf, so they belong to a same box $B=C-I$ of cubic type. By continuity, there exists a small box $x \in B^{\prime}=C-J$ such that the closures of $B^{\prime}, f\left(B^{\prime}\right)$ are in $B$. Let $f_{2}$ be the map given by Lemma 6.3. and let $f_{1}=f \circ f_{2}^{-1}$. It is straightforward to check they satisfy the conditions of the lemma.

Next we need a topological lemma on one dimensional tilable laminations. If $B=C-(a, b)$ is a box of cubic type, for an element $x \in C-b$, its return time to $C-a$ is

$$
\tau_{C-a}(x)=\inf \{t>0 ; x-t \in C-a\} .
$$

By minimality, $\tau_{C-a}(x)$ is finite for any $x \in C-b$, and the map $\tau_{C-a}: C-b \rightarrow \mathbb{R}$ is locally constant, hence continuous.

Lemma 6.5. Let $\Omega$ be a $\mathbb{R}$-tilable lamination, and let $B=C-(a, b)$ be a box of cubic type. Then the following map is an homeomorphsim.

$$
\begin{aligned}
\left\{(x, t) ; x \in C-b, 0 \leq t \leq \tau_{C-a}(x)\right\} & \longrightarrow \Omega \backslash B \\
(x, t) & \mapsto x-t .
\end{aligned}
$$


The proof is plain.

of Theorem 6.1. Let us denote by $H$ the group $\operatorname{Homeo}_{\mathcal{L}}^{0}(\Omega)$ or $\operatorname{Homeo}_{v s p}^{0}(\Omega)$ and let $f \in H$. Let $f_{1}$ and $f_{2}$ be the homeomorphims in $H$ and $B, B^{\prime}$ be the boxes given by Lemma 6.4. From Lemma 6.5 applied to the box $B^{\prime}=C-(a, b)$, and since the map $\tau_{C-a}$ is locally constant, there exists a clopen partition $\left\{C_{1}, \ldots, C_{\ell}\right\}$ of $C$ such that for any $i, \tau_{C-a} \mid C_{i}$ is constant, equals to $\tau_{i}$ and $\left\{C_{i}-\left[0, \tau_{i}\right]\right\}_{i=1}^{\ell}$ is a covering of $\Omega \backslash B$ by closed boxes with interior pairwise disjoint.

Hence, the map $f_{1}$ preserves any box $C_{i}-\left[0, \tau_{i}\right]$, so it can be written as a product of maps $g_{1} \cdots g_{\ell}$, where any $g_{i} \in H$ and supp $g_{i} \subset C_{i}-\left[0, \tau_{i}\right]$. By Lemma 6.2, $f_{2}$ is a commutator and any $g_{i}$ is a commutator $\left[a_{i}, b_{i}\right]$ where the homeomorphisms $a_{i}, b_{i} \in H$ have their support in the box $C_{i}-\left[0, \tau_{i}\right]$. Since two homeomorphisms with disjoint interior of supports commute, we have

$$
f_{1}=\prod_{i=1}^{\ell} g_{i}=\prod_{i=1}^{\ell}\left[a_{i}, b_{i}\right]=\left[\prod_{i=1}^{\ell} a_{i}, \prod_{i=1}^{\ell} b_{i}\right] .
$$

It follows that $f$ may be written as a product of two commutators.

Acknowledgments. It is a pleasure for S. Petite to acknowledge A. Rivière for all the discussions on the subtleties of the Schoenflies Theorem.

\section{REFERENCES}

[1] J. Aliste-Prieto, Translation numbers for a class of maps arising from one-dimensional quasicrystals. Erg. Theo. Dyn. Sys. (2010) 30 Issue 2 pp.565-594

[2] R. D. Anderson, On homeomorphisms as products of conjugates of a given homeomorphism and its inverse. Topology of 3-manifolds and related topics (Proc. The Univ. of Georgia Institute, 1961) pp. 231-234 Prentice-Hall, Englewood Cliffs, N.J.

[3] J. Bellissard; R. Benedetti;J.-M. Gambaudo, Spaces of tilings, finite telescopic approximations and gap-labeling. Comm. Math. Phys. 261 (2006), no. 1, 1-41.

[4] A. Bounemoura, Simplicité des groupes de transformations de surfaces. Ensaios Matemáticos 14. Sociedade Brasileira de Matemática, Rio de Janeiro, 2008. ii+147 pp.

[5] R. Edwards; R. C. Kirby, Deformations of spaces of imbeddings. Ann. Math. (2) 931971 63-88.

[6] D.B.A. Epstein, The simplicity of certain group of homeomorphisms. Comp. Math. 22, (1970), $165-173$

[7] G. M. Fisher, On the group of all homeomorphisms of a manifold. Trans. Amer. Math. Soc. $971960193-212$.

[8] K. Fukui, Commutator Length of Leaf Preserving Diffeomorphisms. Publications of the Research Institute for Mathematical Sciences 48, Issue 3, 2012, pp. 615-622

[9] M. R. Herman, Sur le groupe des difféomorphismes du tore. Ann. Inst. Fourier (Grenoble) 23, (1973), 75-86.

[10] J. Kellendonk. Pattern-equivariant functions and cohomology. J. Phys. A 36 (2003), no. 21, $5765-5772$.

[11] J. Kwapisz, Topological friction in aperiodic minimal $\mathbb{R}^{m}$-actions. Fund. Math. 207 (2010), no. $2,175-178$.

[12] J.N., Mather, Commutators of diffeomorphisms. Comment. Math. Helv. I 49 (1974) 512-528 ; II 50 (1975) , 33-40; III 60 (1985), 122-124.

[13] N. Priebe Frank, L. Sadun. Fusion tilings with infinite local complexity. Topology Proceedings 43 (2014), 235-276.

[14] M. Rubin. Locally Moving Groups and Reconstruction Problems, Ordered Groups and Infinite Permutation Groups, Kluwer Acad. Publ., Dordrecht, 1996, pp. 121-157.

[15] T. Rybicki, The identity component of the leaf preserving diffeomorphism group is perfect. Monast. Math. 120 (1995), 289-305. 
[16] T. Tsuboi, On the group of foliation preserving diffeomorphisms. Foliations 2005, ed. by P. Walczak et al. World Scientific, Singapore, (2006), 411-430.

[17] T. Tsuboi, On the uniform simplicity of diffeomorphisms groups. Advanced Studies in Pure Math., 52, (2008), Groups of diffeomorphisms, 505-524.

[18] W. Thurston, Foliations and groups of diffeomorphisms. Bull. Amer. Math. Soc. 80, (1974), 304-307.

José Aliste-Prieto

Departamento de Matematicas

Universidad Andres Bello

Republica 220, Santiago, Chile jose.aliste@unab.cl

Samuel Petite

Laboratoire Amiénois de

Mathématique Fondamentale et Appliquée,

CNRS-UMR 7352,

Université de Picardie Jules Verne,

33 rue Saint Leu,

80039 Amiens Cedex, France. samuel.petite@u-picardie.fr 